\title{
Pudendal nerve entrapment syndrome caused by ganglion cysts along the pudendal nerve
}

\author{
Young Je Kim ${ }^{1}$, Du Hwan Kim ${ }^{2}$ \\ ${ }^{1}$ Department of Rehabilitation Medicine, Dongsan Medical Center, Keimyung University School of Medicine, Daegu, Korea \\ ${ }^{2}$ Department of Physical Medicine and Rehabilitation, Chung-Ang University Hospital, Chung-Ang University College of Medicine, Seoul, Korea
}

Received: June 5, 2020

Revised: June 22, 2020

Accepted: June 23, 2020

Corresponding author:

Du Hwan Kim, MD, PhD

Department of Physical Medicine

and Rehabilitation, Chung-Ang

University Hospital, Chung-Ang

University College of Medicine, 102

Heukseok-ro, Dongjak-gu, Seoul

06973, Korea

Tel: +82-2-6299-1881

Fax: +82-2-6298-1866

E-mail: ri-pheonix@hanmail.net
Pudendal nerve entrapment (PNE) syndrome refers to the condition in which the pudendal nerve is entrapped or compressed. Reported cases of PNE associated with ganglion cysts are rare. Deep gluteal syndrome (DGS) is defined as compression of the sciatic or pudendal nerve due to a non-discogenic pelvic lesion. We report a case of PNE caused by compression from ganglion cysts and treated with steroid injection; we discuss this case in the context of DGS. A 77-year-old woman presented with a 3-month history of tingling and burning sensations in the left buttock and perineal area. Ultrasonography showed ganglion cystic lesions at the subgluteal space. Magnetic resonance imaging revealed cystic lesions along the pudendal nerve from below the piriformis to the Alcock's canal and a full-thickness tear of the proximal hamstring tendon. Aspiration of the cysts did not yield any material. We then injected steroid into the cysts, which resolved her symptoms. Steroid injection into a ganglion cyst should be considered as a treatment option for PNE caused by ganglion cysts.

Keywords: Corticosteroids; Ganglion cysts; Nerve entrapment; Pudendal nerve

\section{Introduction}

Pudendal neuralgia is a neuropathic pain that presents in the distribution of pudendal nerve. The pudendal nerve emerges from the sacral plexus nerves (S2 to S4) and carries sensory fibers to genitalia such as the penis, scrotum, clitoris, and labia, and to the motor fibers of the perineum and pelvic floor muscles. Although pudendal neuralgia can be suspected in the presence of perineal pain, it is difficult to determine a cause in the absence of organic lesions on imaging tests [1]. Pudendal nerve entrapment (PNE) syndrome refers to the condition in which the pudendal nerve is entrapped or compressed [2]. Previous studies have reported that most cases of PNE are related to iatrogenic mechanical insult, such as gynecologic or hip surgery, or from incorrect seating position while cycling [3-5]. Only a few cases of organic lesions associated with PNE have been reported [1,2]. Furthermore, there has only been one published report of PNE related to ganglion cysts; this case was successfully treated with ultrasound-guided aspiration of the cysts [2].

Pudendal neuralgia caused by PNE presents with unilateral pelvic pain. This pain is typically aggravated by a seated position because the pudendal nerve is compressed between the sacrospinous and sacrotuberous ligaments and relieved by sitting on the toilet seat [1]. PNE may also cause pain in the posterior hip, the buttocks while seated, or in the medial thigh; referred sciatic pain has also been reported [1]. Recently, the concept of deep gluteal syndrome (DGS) has been introduced and defined as compression of the sciatic or pudendal nerve due to a non-discogenic pelvic lesion [6]. Thus, PNE can be considered to be part of the DGS spectrum.

In this report, we present a case of PNE caused by ganglion cysts that was successfully treated with steroid injections. We discuss this case in the context of DGS. 


\section{Case}

This case report was approved by the Institutional Review Board of Keimyung University Dongsan Hospital (IRB No: 2020-06037). Written informed consent was obtained from the patient for publication of this case report and accompanying images.

A 77-year-old woman presented to our clinic with a 3-month history of tingling and burning sensations in the left buttock and perineal area. The pain was aggravated in the sitting position and relieved while standing or in a supine position. She had no sphincter dysfunction such as urination or defecation and no history of trauma or pelvic surgery. On physical examination, passive external rotation and abduction of her hip joint produced the inguinal and buttock pain. There was a tenderness over the ischial tuberosity and piriformis area. There was no weakness of the lower extremities except for the left knee flexor (Medical Research Council grade IV). Deep tendon reflexes of the lower limbs were normal. There was no sensory impairment in the left lower extremity or the S4 and S5 dermatomes. Her pedal pulses were normal. We performed an ultrasound to identify the cause of her buttock and perineal pain. Ultrasonography revealed cystic lesions at the subgluteal space and a near full-thickness tear of the proximal hamstring tendons at the ischial tuberosity (Fig. 1). Pelvis magnetic resonance imaging showed ganglion cystic lesions along the pudendal nerve from below the piriformis to the Alcock's canal (Figs. $2,3)$. In addition, we noted fluid collection related to the tear of the hamstring origin sites around the ischial tuberosity (Figs. 2, 3). Based on the proximity of the ganglion cysts to the pudendal nerve, we determined that the cysts caused the PNE. Aspiration of the cysts under ultrasound guidance did not produce material. We then injected steroid (triamcinolone acetonide $20 \mathrm{mg}+1 \%$ lidocaine $3 \mathrm{~mL}$ ) into the cysts, which resolved her burning sensations.

\section{Discussion}

The prevalence of pudendal neuralgia caused by PNE has not been established, mostly due to the fact that it is often underdiagnosed. Kaur and Singh [7] and Spinosa et al. [8] reported an incidence of $1 \%$ in the general population and it is also known to be more common in females than males. While PNE can significantly impair patients' quality of life, owing to its rarity and difficulty of diagnosis, it is often inappropriately treated [7]. Further, the mean time to diagnosis is 4 years and there is no specific diagnostic test [9]. The 'Nantes criteria,' created to facilitate the diagnosis of PNE, include (1) pain in the anatomical territory of the pudendal nerve, (2) pain that is worsened by sitting, (3) the patient is not woken at night by the pain, (4) there is no objective sensory loss on clinical examination, and (5) pain relief provided by an anesthetic pudendal nerve block [10]. In our case, the patient met the first four diagnostic criteria.

The pudendal nerve originates from the S2, S3, and S4 sacral nerve roots and courses between the piriformis and ischiococcygeus muscles, leaving the pelvis through the greater sciatic foramen. It then crosses the sacrospinous ligament and re-enters the pelvis through the lesser sciatic foramen. After re-entrance, it courses anterosuperiorly through the Alcock's canal. The nerve divides into three branches as it exits the canal. The Alcock's canal lies on the medial surface of the obturator internus muscle and the medial aspect of ischial tuberosity above the falciform ridge $[7,11]$. In our patient, ganglion cysts were observed along the pudendal nerve from below the piriformis to the Alcock's canal. Cystic lesions observed along the pudendal nerve should be distinguished from extraneural or intraneural ganglion cysts. Because our patient did not receive an exploratory operation or high-resolution magnetic resonance neurography, the characteristics of the cysts were not identified in detail.

DGS encompasses a variety of conditions including piriformis, gemelli-obturator internus, ischiofemoral impingement, and proximal hamstring syndromes. The presenting symptoms can be localized in the perineal, perianal and posterior areas, with the specific location depending on which nerve is compressed or irritated. Previous studies of DGS have mainly discussed involvement of the sciatic nerve rather than of the pudendal nerve. Hamstring tears around the ischial tuberosity can also be a cause of DGS. Further, proximal hamstring tendon pathologies can cause a ganglion cyst that irritates or compresses the adjacent nerves [6]. In our case, ganglion cysts along the pudendal nerve seemed to be associated with a full-thickness tear of the proximal hamstring tendon. We consider our patient's symptoms to fit into the category of DGS.

Ganglion cysts have been reported in most joints, tendons, and, rarely, in bone. On average, they have a 50\% spontaneous resolution rate. While the management of ganglion cysts ranges from nonsurgical to surgical treatments, aspiration is the mainstay of nonsurgical treatment [12]. Lee et al. [2] reported the first case of PNE caused by a ganglion cyst and suggested ultrasound-guided aspiration as a treatment option to alleviate pain. However, the recurrence rate of cysts treated with aspiration alone is more than $50 \%$ [12]. To decrease recurrence after simple aspiration, steroid injections have been used [13]. In our patient's case, aspiration of the cysts was non-productive but an injected steroid was successful in alleviating her pain. Considering that aspiration of the cyst did not produce material, the injected steroid might spread to the 

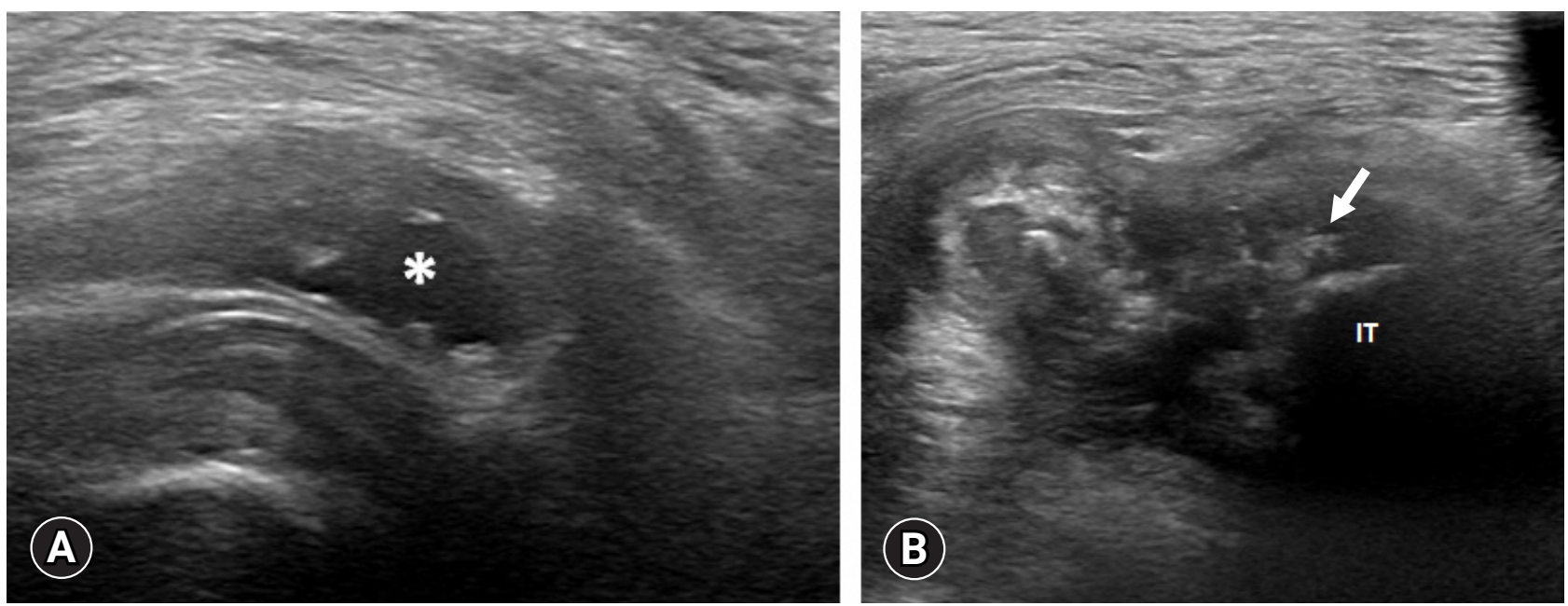

Fig. 1. Ultrasonographic findings. (A) A 2.6x1.2 cm-sized ganglion cystic lesion (asterisk) at the subgluteal space. (B) Full-thickness tear (arrow) of the proximal hamstring tendon at the ischial tuberosity (IT).
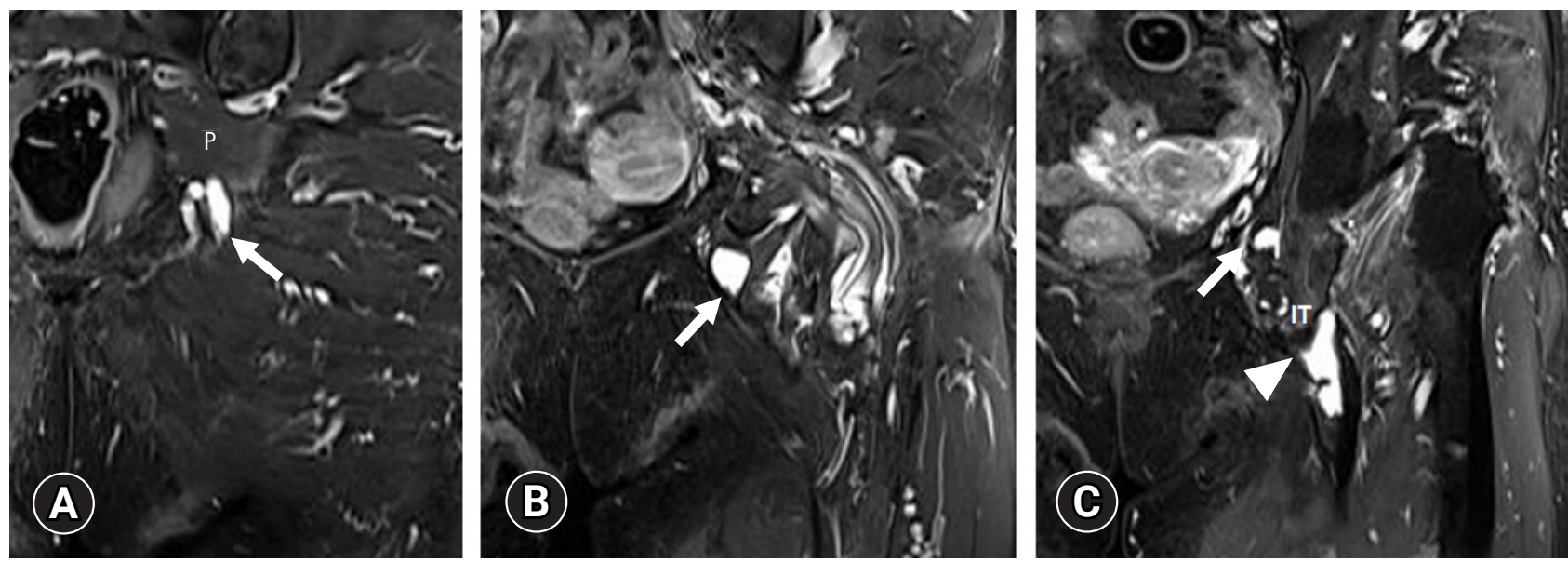

Fig. 2. Coronal fat-suppressed T2-weighted images of ganglion cyst courses along the pudendal nerve. (A) A $2.0 \times 0.8 \mathrm{~cm}$-sized ganglion cyst (arrow) below piriformis (P). (B) A 1.8x1.4 cm-sized ganglion cyst (arrow) at the entrance of the Alcock's canal. (C) A 1.0x0.75 cmsized ganglion cyst (arrow) within the Alcock's canal and full-thickness tear (arrowhead) of the proximal hamstring tendon at the ischial tuberosity (IT).
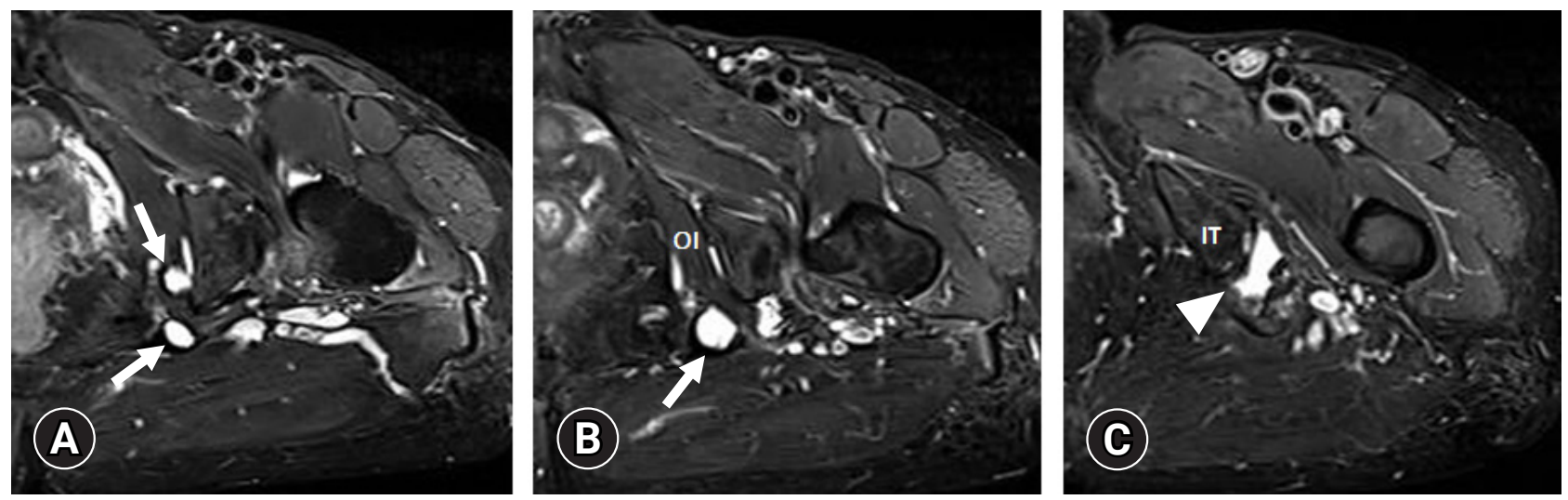

Fig. 3. Axial fat-suppressed T2-weighted images of ganglion cyst courses along the pudendal nerve. (A) A $1.0 \times 0.8 \mathrm{~cm}$-sized and $1.2 \times 1.3$ $\mathrm{cm}$-sized ganglion cysts (arrows) around the Alcock's canal. (B) A $1.3 \times 1.5 \mathrm{~cm}$-sized ganglion cyst (arrow) medial to the obturator internus (OI) muscle. (C) A full-thickness tear (arrowhead) of the proximal hamstring tendon at the ischial tuberosity (IT). 
pudendal nerve and lead to a significant improvement, such as perineural steroid injection in PNE patients without ganglion cyst [9]. Steroids might suppress ectopic nerve activity from damaged peripheral nerve fibers and stabilize the nerve's membrane [14]. This suggests that steroid injection should be considered as a treatment option for patients with PNE in whom aspiration of ganglion cysts to alleviate the pain has failed.

In conclusion, in patients with unilateral perineal and buttock area pain, PNE should be considered as a possible cause of the pain. We suggest steroid injections as a treatment option for PNE associated with ganglion cysts, particularly when aspiration is unsuccessful.

\section{Acknowledgments}

\section{Conflicts of interest}

No potential conflict of interest relevant to this article was reported.

\section{Author contributions}

Conceptualization, Formal analysis, Supervision, and Writing-review \& editing: DHK; Data curation, Writing-original draft: YJK.

\section{ORCID}

Young Je Kim, https://orcid.org/0000-0001-6350-7675

Du Hwan Kim, https://orcid.org/0000-0002-9980-8549

\section{References}

1. Ploteau S, Cardaillac C, Reina MA, Perrouin-Verbe E, Riant T, Labat JJ. Pudendal neuralgia due to pudendal nerve entrapment: warning signs observed in two cases and review of the literature. Pain Physician 2016;19:E449-54.

2. Lee JW, Lee SM, Lee DG. pudendal nerve entrapment syndrome due to a ganglion cyst: a case report. Ann Rehabil Med 2016;40:741-4.

3. Alevizon SJ, Finan MA. Sacrospinous colpopexy: management of postoperative pudendal nerve entrapment. Obstet Gynecol 1996;88(4 Pt 2):713-5.

4. Pailhe R, Chiron P, Reina N, Cavaignac E, Lafontan V, Laffosse JM. Pudendal nerve neuralgia after hip arthroscopy: retrospective study and literature review. Orthop Traumatol Surg Res 2013;99:785-90.

5. Leibovitch I, Mor Y. The vicious cycling: bicycling related urogenital disorders. Eur Urol 2005;47:277-87.

6. Park JW, Lee YK, Lee YJ, Shin S, Kang Y, Koo KH. Deep gluteal syndrome as a cause of posterior hip pain and sciatica-like pain. Bone Joint J 2020;102-B:556-67.

7. Kaur J, Singh P. Pudendal nerve entrapment syndrome [Internet]. Treasure Island (FL): StatPearls Publishing; 2020 [cited 2020 Jun 2].https://www.ncbi.nlm.nih.gov/books/NBK544272/.

8. Spinosa JP, de Bisschop E, Laurencon J, Kuhn G, Dubuisson JB, Riederer BM. Sacral staged reflexes to localize the pudendal compression: an anatomical validation of the concept. Rev Med Suisse 2006;2:2416-8, 2420-1.

9. Itza Santos F, Salinas J, Zarza D, Gomez Sancha F, Allona Almagro A. Update in pudendal nerve entrapment syndrome: an approach anatomic-surgical, diagnostic and therapeutic. Actas Urol Esp 2010;34:500-9.

10. Labat JJ, Riant T, Robert R, Amarenco G, Lefaucheur JP, Rigaud J. Diagnostic criteria for pudendal neuralgia by pudendal nerve entrapment (Nantes criteria). Neurourol Urodyn 2008;27:306-10.

11. Montoya TI, Calver L, Carrick KS, Prats J, Corton MM. Anatomic relationships of the pudendal nerve branches. Am J Obstet Gynecol 2011;205:504.

12. Gude W, Morelli V. Ganglion cysts of the wrist: pathophysiology, clinical picture, and management. Curr Rev Musculoskelet Med 2008;1:205-11.

13. Derbyshire RC. Observations on the treatment of ganglia with a report on hydrocortisone. Am J Surg 1966;112:635-6.

14. Devor M, Govrin-Lippmann R, Raber P. Corticosteroids suppress ectopic neural discharge originating in experimental neuromas. Pain 1985;22:127-37. 